\title{
Essai d'utilisation de la bêta-D-galactosidase pour la fabrication du lait condensé sucré
}

\author{
par
}

\author{
J. KISZA, J. SWITKA, A. KRUK et A. SURAZYNSKI \\ Institut du Génie et de la Biotechnologie alimentaire \\ de l'Université agricole d'Olsztyn (Pologne)
}

\section{INTRODUCTION}

La cristallisation du lactose représente l'étape la plus difficile dans la production du lait condensé sucré. La formation de cristaux de lactose, aux dimensions de $10-50 \mu$ et davantage, contribue aux défauts de structure sableuse et farineuse dans le produit fini. Selon Sparks [7], les dimensions des cristaux de lactose dans le lait condensé sucré à structure lisse et régulière ne doivent pas dépasser $10 \mu$, et le nombre des cristaux ayant de telles dimensions doit atteindre au moins $300000 / \mathrm{mm}^{3}$. Par contre, Hunziker [3] rapporte, que le lait condensé sucré ayant une structure lisse contient 400000 cristaux dans $1 \mathrm{~mm}^{3}$, ce qui correspond à une longueur de l'axe du cristal de $9 \mu$ environ.

Le processus de cristallisation du lactose, qui se déroule dans un milieu aussi hétérogène que le lait, est bien compliqué, conditionné par des facteurs multiples, tels que la vitesse de mutarotation des formes de $\alpha$ et $\beta$-lactose, le degré de saturation de la solution, la teneur en sucrose, la valeur du $\mathrm{pH}$, la viscosité et la température du lait condensé.

Ayant en vue les difficultés qu'on rencontre au cours de la cristallisation régulière dans le lait condensé, la tentation est grande d'utiliser l'enzyme de la $\beta$-D-Galactosidase, produite par la levure Saccharomyces fragilis, pour hydrolyser une partie du lactose. Un autre moyen d'utiliser to latose, non moins important, est de l'employer pour prévenir la cristallisation dans la crême glacée et autres produits laitiers congelés, ainsi que pour la fabrication d'aliments pour enfants qui ne tolèrent pas le lactose [8].

\section{PROTOCOLE EXPERIMENTAL}

Les expérimentations ont été effectuées en deux temps : dans le premier, des conditions optima de l'hydrolyse enzymatique du lactose étaient établies, tandis que, dans le second, le lait condensé sucré 
était fabriqué avec du lactose partiellement hydrolysé dans des conditions de cristallisation bien précisées.

Le lait entier, d'une teneur en matière grasse de 3,2 p. 100 a servi de matière première dans nos recherches. Après pasteurisation à $92^{\circ} \mathrm{C}$ pendant $2 \mathrm{mn}$, le lait était ensemencé avec l'enzyme, pour être ensuite refroidi jusqu'à température d'incubation, à laquelle le processus d'hydrolyse du lactose se développait. Dans la première série de cette étape de l'expérimentation, trois températures d'incubation étaient appliquées, à savoir :

$37^{\circ} \mathrm{C}$ pendant $4 \mathrm{~h}$,

$15^{\circ} \mathrm{C}$ pendant $15 \mathrm{~h}$,

$8^{\circ} \mathrm{C}$ pendant $15 \mathrm{~h}$.

Dans tous les cas, on ajoutait 0,1 p. 100 de la préparation de $\beta$-D-galactosidase qui avait été obtenue à partir de la levure Saccharomyces fragilis. Dans la seconde série expérimentale, deux températures seulement ont été appliquées, c'est-à-dire $37^{\circ} \mathrm{C}$ pendant $4 \mathrm{~h}$ et $8^{\circ} \mathrm{C}$ pendant $15 \mathrm{~h}, 0,1$ p. 100 d'enzyme étant ajouté au lait à $37^{\circ} \mathrm{C}$, et le lait étant continuellement agité au cours de l'incubation.

La seconde étape de l'expérimentation a été réalisée, elle aussi, sur le plan du laboratoire. Au total, 9 fabrications ont été faites à partir du lait, dans lequel le lactose avait été partiellement décomposé par la $\beta$-D-galactosidase. Par comparaison, une fabrication a été faite à partir du lait qui n'avait pas subi d'hydrolyse du lactose. Le procédé de fabrication du lait condensé sucré était effectué d'après le schéma suivant :

Le lait normalisé était d'abord pasteurisé à $92^{\circ} \mathrm{C}$ pendant $2 \mathrm{mn}$, puis refroidi jusqu'à $37^{\circ} \mathrm{C}$ et divisé en deux parties. L'une était additionnée à 0,1 p. 100 d'enzyme $\beta$-D-galactosidase, refroidie jusqu'à $8^{\circ} \mathrm{C}$ et incubée à cette température pendant $15 \mathrm{~h}$. L'autre lot, non hydrolysé, était maintenu aux mêmes températures. Après $15 \mathrm{~h}$, le lait des deux lots était mélangé en proportions de $1 / 1$ ou $2 / 1$ et pasteurisé de nouveau pour inactiver l'enzyme.

Le sucrose était introduit dans le lait sous forme de sirop à 75 p. 100. Le processus de condensation était réalisé dans un évaporateur de laboratoire à $50^{\circ} \mathrm{C}-55^{\circ} \mathrm{C}$ sous pression de $500-560 \mathrm{~mm} \mathrm{Hg}$ jusqu'à 72,5 p. 100 de teneur en extrait sec.

Le produit fini était divisé en deux parties, l'une étant vite refroidie (pendant $1 \mathrm{~h}$ avec de l'eau courante à $10^{\circ} \mathrm{C}-20^{\circ} \mathrm{C}$ ), tandis que l'autre était exposée au refroidissement spontané (pendant $5 \mathrm{~h}$ ), jusqu'à la même température. Les deux lots de lait condensé, ainsi refroidis, étaient maintenus à la même température jusqu'au moment de l'analyse.

\section{METHODES}

En la matière, les déterminations suivantes ont été effectuées : acidité active et potentielle [1], degré d'alcool [1, 4], teneur en lactose, par la méthode de Bertrand, avant et après l'hydrolyse. 
TABLEAU 1

Conditions de l'hydrolyse du lactose, effectuée dans le lait par la bêta-D-galactosidase ( 0,1 p. 100 de préparation ajouté)

\begin{tabular}{|c|c|c|c|c|c|c|c|c|c|c|c|c|}
\hline \multirow{3}{*}{$\begin{array}{l}\text { Température } \\
\text { d'incubation }\end{array}$} & \multicolumn{4}{|c|}{$37^{\circ} \mathrm{C}$} & \multicolumn{4}{|c|}{$15^{\circ} \mathrm{C}$} & \multicolumn{4}{|c|}{$8^{\circ} \mathrm{C}$} \\
\hline & \multirow{2}{*}{$\begin{array}{c}\text { Temps } \\
\text { d'hydrolyse } \\
\text { heures }\end{array}$} & \multicolumn{2}{|c|}{ Acidité } & \multirow{2}{*}{$\begin{array}{l}\text { Degré de } \\
\text { l'hydrolyse } \\
\text { de lactose }\end{array}$} & \multirow{2}{*}{$\begin{array}{c}\text { Temps } \\
\text { d'hydrolysè } \\
\text { heures }\end{array}$} & \multicolumn{2}{|c|}{ Acidité } & \multirow{2}{*}{$\begin{array}{c}\text { Degré de } \\
\text { l'hydrolyse } \\
\text { de lactose }\end{array}$} & \multirow{2}{*}{$\begin{array}{c}\text { Temps } \\
\text { d'hydrolyse } \\
\text { heures }\end{array}$} & \multicolumn{2}{|c|}{ Acidité } & \multirow{2}{*}{$\begin{array}{l}\text { Degré de } \\
\text { l'hydrolyse } \\
\text { de lactose }\end{array}$} \\
\hline & & $\mathrm{pH}$ & $\mathrm{SH}$ & & & $\mathrm{pH}$ & $\mathrm{SH}$ & & & $\mathrm{pH}$ & $\mathrm{SH}$ & \\
\hline I & $\begin{array}{l}0 \\
1 \\
2 \\
3 \\
4\end{array}$ & $\begin{array}{l}6,62 \\
6,60 \\
6,60 \\
6,58 \\
6,50\end{array}$ & $\begin{array}{l}7,2 \\
7,2 \\
7,2 \\
7,2 \\
7,8\end{array}$ & $\begin{array}{l}\overline{10,4} \\
21,0 \\
31,3 \\
34,5\end{array}$ & $\begin{array}{l}0 \\
2,5 \\
5 \\
10 \\
15\end{array}$ & $\begin{array}{l}6,62 \\
6,60 \\
6,60 \\
6,60 \\
6,60\end{array}$ & $\begin{array}{l}7,0 \\
7,2 \\
7,2 \\
7,6 \\
7,6\end{array}$ & $\begin{array}{l}-\overline{10,4} \\
15,7 \\
31,5 \\
36,8\end{array}$ & $\begin{array}{l}0 \\
2,5 \\
5 \\
10 \\
15\end{array}$ & $\begin{array}{l}6,62 \\
6,62 \\
6,60 \\
6,60 \\
6,60\end{array}$ & $\begin{array}{l}7,0 \\
7,0 \\
7,0 \\
7,0 \\
7,0\end{array}$ & $\begin{array}{r}\overline{7,8} \\
15,7 \\
18,4 \\
24,7\end{array}$ \\
\hline II & $\begin{array}{l}0 \\
1 \\
2 \\
3 \\
4\end{array}$ & $\begin{array}{l}6,75 \\
6,70 \\
6,68 \\
6,68 \\
6,65\end{array}$ & $\begin{array}{l}7,0 \\
7,0 \\
7,2 \\
7,2 \\
7,6\end{array}$ & $\begin{array}{l}-\overline{21,0} \\
31,5 \\
34,7 \\
42,1\end{array}$ & $\begin{array}{l}- \\
- \\
-\end{array}$ & $\begin{array}{l}- \\
- \\
- \\
-\end{array}$ & $\begin{array}{l}- \\
- \\
-\end{array}$ & $\begin{array}{l}- \\
z \\
-\end{array}$ & $\begin{array}{l}0 \\
2,5 \\
5 \\
10 \\
15\end{array}$ & $\begin{array}{l}6,75 \\
6,75 \\
6,70 \\
6,70 \\
6,70\end{array}$ & $\begin{array}{l}7,0 \\
7,0 \\
7,2 \\
7,2 \\
7,2\end{array}$ & $\begin{array}{r}-\overline{18,4} \\
25,8 \\
25,8 \\
29,0\end{array}$ \\
\hline
\end{tabular}


Dans le produit fini, on a déterminé l'acidité active et potentielle - titrable - [1], la teneur en extrait sec, en lactose et en sucrose. L'examen organoleptique, ainsi que des observations et des mesures des cristaux de lactose étaient aussi effectuées, en adoptant un grossissement de 620 fois.

\section{RESULTATS ET DISCUSSION}

Selon son origine et la qualité du matériel d'étude employé et des conditions d'hydrolyse appliquées, la $\beta$-D-galactosidase peut montrer une activité diverse $[2,6]$. De nombreux auteurs montrent que l'activité la plus élevée était observée dans la solution tamponnée de lactose additionnée d'ions $\mathrm{Na}, \mathrm{K}$ et $\mathrm{Mn}(6,9)$. Dans des milieux naturels, à partir des composants du lait (lait entier ou écrémé, lactosérum), l'activité du lactose la plus élevée était constatée dans le lactosérum, tandis que celle du lait entier s'est montrée la moins élevée.

C'est bien là une des raisons qui nous a fait étudier l'intensité de l'hydrolyse du lactose en fonction de la température d'incubation. L'application de températures diverses $\left(37^{\circ} \mathrm{C}, 15^{\circ} \mathrm{C}\right.$ et $\left.8^{\circ} \mathrm{C}\right)$ avait pour but de démontrer pour quels paramètres la $\beta$-D-galactosidase peut atteindre le degré suffisamment élevé d'hydrolyse de lactose, sans avoir produit des changements indésirables dans l'acidité et les propriétés organoleptiques du lait.

Nos essais démontrent que l'addition de 0,1 p. 100 de la préparation de $\beta$-D-galactosidase donnait un degré d'hydrolyse du lactose suffisamment élevé. Les données du tableau 1 indiquent que l'intensité de cette réaction diminuait avec la température, ce qui se manifestait d'une manière la plus évidente pour une amplitude de température de $8^{\circ} \mathrm{C}-15^{\circ} \mathrm{C}$. Le même tableau montre que les effets d'hydrolyse de la seconde série étaient meilleurs. On a pourtant omis la température de $15^{\circ} \mathrm{C}$, surtout en raison de la durée trop prolongée de l'hydrolyse et la possibilité des arrière-goûts étrangers (de malt, de levure), ce qui avait été constaté dans la première série, au cours de l'hydrolyse à $15^{\circ} \mathrm{C}$ et $37^{\circ} \mathrm{C}$. Nos observations ne sont pas toutes d'accord avec les résultats d'Albreht et Gracy [6] qui ont, en principe, établi l'influence avantageuse de l'hydrolyse du lactose à $37^{\circ} \mathrm{C}$, pendant une très courte durée, sans avoir constaté des effets accessoires, tels que l'altération des caractéristiques de goûts et d'odeur du lait hydrolysé.

La disposition et l'allure des courbes des diagrammes 1 et 2 montrent que l'activité de l'enzyme en question devient la plus élevée pendant les premières heures de l'hydrolyse, une petite quantité des monosaccharides se trouvant encore dans le milieu. Nos suggestions concernant une corrélation entre le ralentissement de l'hydrolyse du 
lactose et l'augmentation des monosaccharides ont été confirmées par les travaux de Pomeranz [6] et de Wendorf [9].

L'analyse des résultats présentés jusqu'à maintenant nous a permis de choisir et d'appliquer, dans la seconde étape de l'expérimentation, les conditions les plus favorables pour l'hydrolyse, à savoir : 0,1 p. 100 d'enzyme introduite dans le lait à $37^{\circ} \mathrm{C}$, suivi immédiatement par un refroidissement jusqu'à $8^{\circ} \mathrm{C}$ et l'incubation à la même température pendant $15 \mathrm{~h}$. Ce système s'est montré avantageux, également du point de vue de l'organisation.

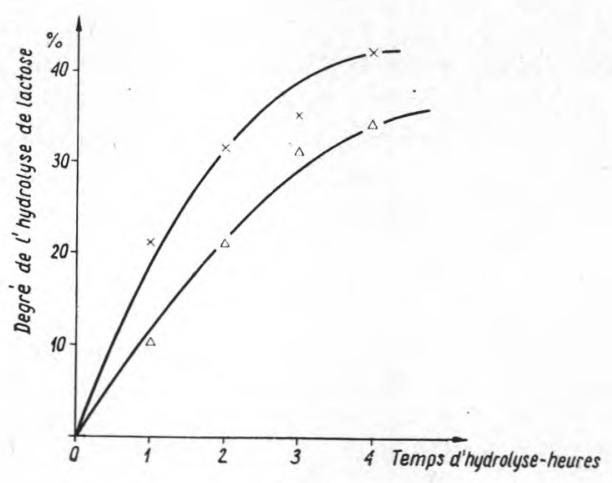

fig. 1

Degré d'hydrolyse du lactose en fonction de la durée d'hydrolyse à $37^{\circ} \mathrm{C}$

$\triangle \longrightarrow$ Série I

$\times \quad$ Série II

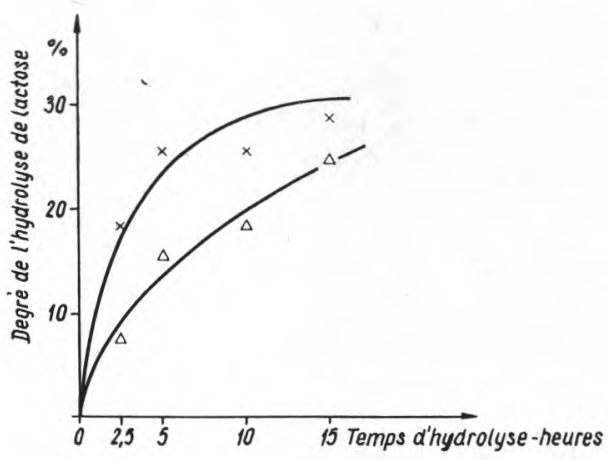

fig. 2

Degré d'hydrolyse du lactose en fonction de la durée d'hydrolyse à $8^{\circ} \mathrm{C}$

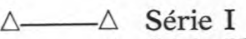

$\times$ Série II 
TABLEAU 2

Caractéristique chimique de la matière première et du produit fini (valeurs moyennes)

\begin{tabular}{|c|c|c|c|c|c|c|c|c|c|c|}
\hline \multirow{3}{*}{ Sorte de fabrication } & \multicolumn{5}{|c|}{ Matière première } & \multicolumn{5}{|c|}{ Produit fini } \\
\hline & \multicolumn{2}{|c|}{ Acidité } & \multirow{2}{*}{$\begin{array}{c}\text { Taux } \\
\text { d'alcool } \\
\text { ml }\end{array}$} & \multicolumn{2}{|c|}{ Teneur en lactose } & \multicolumn{2}{|c|}{ Acidité } & \multirow{2}{*}{$\begin{array}{c}\text { Teneur en } \\
\text { E.S. } \\
\text { p. } 100\end{array}$} & \multicolumn{2}{|c|}{ Teneur en lactose } \\
\hline & $\mathrm{pH}$ & $\circ \mathrm{SH}$ & & $\begin{array}{l}\text { Total } \\
\text { p. } 100\end{array}$ & $\begin{array}{c}\text { Degré } \\
\text { d'hydrolyse }\end{array}$ & $\mathrm{pH}$ & ${ }^{\circ} \mathrm{SH}$ & & $\begin{array}{l}\text { Total } \\
\text { p. } 100\end{array}$ & $\begin{array}{l}\text { Dans l'eau du } \\
\text { lait condensé }\end{array}$ \\
\hline Témoin & 6,45 & 6,8 & 8,5 & 4,0 & - & 5,80 & 14,0 & 72,2 & 9,3 & 25,1 \\
\hline $1: 1$ & 6,62 & 7,1 & 8,3 & 3,65 & 11,1 & 6,00 & 13,4 & 72,7 & 8,45 & 23,6 \\
\hline $2: 1$ & 6,54 & 7,2 & 7,8 & 3,50 & 14,1 & 6,00 & 15,2 & 72,6 & 8,1 & 22,8 \\
\hline
\end{tabular}


Avant le processus de condensation, le lait hydrolysé était mélangé avec du lait non hydrolysé, à raison de $1 / 1$ ou $2 / 1$. Cette opération avait pour but de différencier la teneur en lactose du lait de transformation, ainsi que de vérifier la possibilité d'utiliser, dans le procédé de fabrication, de petites quantités de lait hydrolysé, présentant pourtant un degré élevé d'hydrolyse du lactose.

L'évaluation de la matière première ainsi composée nous a permis de constater que les changements d'acidité potentielle et active dus aux préparations du lait pour la fabrication ne sont pas grands. Il apparaît que ces changements ne sont pas essentiels du point de vue pratique, parce que le lait, préparé de la sorte, peut être employé, avec effet satisfaisant, pour la fabrication du lait condensé sucré. De même, la diminution du degré d'alcool, qui dépend strictement des variations d'acidité, ne disqualifiait point ce milieu pour la fabrication des concentrés (tab. 2).

Le tableau 2 présente aussi les résultats des déterminations de la teneur en lactose du lait de transformation, ainsi que celle du lait condensé sucré qui en était obtenu. Ces valeurs diminuent à mesure que la quantité du lait hydrolysé ajouté augmente. Les valeurs les plus élevées ont été obtenues dans du lait concentré qui avait été fabriqué sans addition de lait hydrolysé. La teneur en lactose était de 9,3 p. 100 , ce qui, calculé en teneur en lactose de l'eau du lait condensé, donne une valeur de 25,1 p. 100. Par contre, dans le condensé fabriqué avec du lait hydrolysé ajouté, ces valeurs se maintiennent à un niveau bien moins élevé, c'est-à-dire 8,1 p. 100 et 8,45 p. 100 ainsi que 22,8 et 23,6 p. 100 respectivement.

On sait que la solubilité du lactose dans l'eau à température ambiante, est de 18 p. 100 environ, eu égard à l'équilibre de $\alpha$ et $\beta$-lactose. Ainsi, tout l'excès de lactose contenu dans le concentré peut cristalliser, et la forme et les dimensions des cristaux de lactose ne sont point indifférents pour la qualité des produits finis.

Les observations que nous avons faites, ainsi que les mesures microscopiques de la grandeur des cristaux de lactose, ont montré que, dans l'échantillon témoin du lait condensé sucré, 40-50 p. 100 du lactose cristallisé avait la forme des cristaux aux dimensions de plus de $10 \mu$ (tab. 3 ). Ce produit montrait une texture sableuse très nette. Ce défaut de texture farineuse et légèrement sableuse était aussi présent dans des produits faits à partir de la matière première avec une quantité moins élevée de lait hydrolysé ajouté, et surtout dans le lot du produit fini qui avait subi le refroidissement spontané. En ce cas, le degré d'hydrolyse du lactose dans le lait de transformation était de 11,1 p. 100 , la concentration de lactose dans l'eau du concentré était de 23,6 p. 100 et la proportion des cristaux qui dépassait $10 \mu$ atteignait 28,3 p. 100 . Dans les autres produits, indépendamment de la méthode de leur refroidissement, le nombre observé des grands cristaux de lactose n'était pas élevé, les formes inférieures à $10 \mu$ constituant 92 p. 100 du nombre total de cristaux. Comme il 
$T A B L E A U 3$

Résultats de l'examen organoleptique, eu égard aux dimensions des cristaux de lactose présents

\begin{tabular}{|c|c|c|c|c|c|c|}
\hline \multirow{2}{*}{$\begin{array}{c}\text { Sorte } \\
\text { de } \\
\text { fabrication }\end{array}$} & \multirow{2}{*}{$\begin{array}{l}\text { Manière } \\
\text { de } \\
\text { refroidir }\end{array}$} & \multicolumn{2}{|c|}{$\begin{array}{l}\text { Distribution des cristaux } \\
\text { d'après leurs dimensions }\end{array}$} & \multicolumn{3}{|c|}{ Examen organoleptique } \\
\hline & & $\begin{array}{l}\text { jusqu'à } 10 \mu \\
\text { p. } 100\end{array}$ & $\begin{array}{l}\text { plus de } 10 \mu \\
\text { p. } 100\end{array}$ & Goût et odeur & Couleur & Texture \\
\hline Témoin & $\mathrm{a}$ & $\begin{array}{l}60,8 \\
47,1\end{array}$ & $\begin{array}{l}39,2 \\
52,9\end{array}$ & $\begin{array}{c}\text { Typique, régulier } \\
»\end{array}$ & $\begin{array}{c}\text { Crème } \\
\text { » }\end{array}$ & $\begin{array}{c}\text { Dense, nette, texture } \\
\text { sableuse } \\
»\end{array}$ \\
\hline $1: 1$ & $\begin{array}{l}a \\
b\end{array}$ & $\begin{array}{l}92,0 \\
71,7\end{array}$ & $\begin{array}{r}8,0 \\
28,3\end{array}$ & $\begin{array}{c}\text { Typique, régulier } \\
»\end{array}$ & $\begin{array}{c}\text { Crème - jaune } \\
»\end{array}$ & $\begin{array}{l}\text { Dense, texture sableuse } \\
\text { légèrement perceptible }\end{array}$ \\
\hline $2: 1$ & $\begin{array}{l}a \\
b\end{array}$ & peu nc & breux & $\begin{array}{c}\text { Typique, régulier } \\
»\end{array}$ & $\begin{array}{c}\text { Jaune } \\
»\end{array}$ & $\begin{array}{c}\text { Dense, aucune } \\
\text { perception des cristaux }\end{array}$ \\
\hline a : produit c & subi le & idissement $f$ & & $\mathrm{~b}$ : produit re & pontanément & \\
\hline
\end{tabular}


ressort de l'examen organoleptique, le défaut de texture sableuse et farineuse n'est pas dû à ces petits cristaux.

Comme il ressort du tableau 3, la méthode de refroidissement de produit après sa fabrication influence d'une manière essentielle le nombre et la grandeur des cristaux de lactose. Les échantillons de lait condensé qui avaient subi le refroidissement rapide contenaient bien moins de cristaux montrant des dimensions supérieures à $10 \mu$. Le refroidissement rapide contribue à la formation d'un grand nombre de centres de cristallisation, et l'augmentation de la viscosité qui en résulte diminue la vitesse de diffusion des molécules de lactose vers les centres de cristallisation formés. Ainsi, l'effet final de la cristallisation est une résultante de tous les paramètres participant à ce processus.

Les résultats, obtenus à l'examen organoleptique, permettent de constater que tous les échantillons de lait condensé sucré avaient un goût et une odeur normaux. On n'a observé aucune altération de couleur, bien que la teneur élevée en mono-saccharides y put apparaître comme une circonstance favorable. Dluzewski [2] et Pomeranz [6] ont pris en considération une telle possibilité. D'après eux, le changement de couleur est dû à la formation des combinaisons de Maillard, surtout au cours du processus prolongé de la concentration aux températures élevées.

\section{CONCLUSIONS}

1) L'analyse générale de nos résultats nous permet de constater, que la $\beta$-D-galactosidase peut être utilisée dans la fabrication du lait condensé pour y éviter le défaut de texture sableuse.

2) Pour atteindre cet objectif, il suffit de décomposer 15 p. 100 environ du lactose présent dans le lait, sans que la teneur en lactose de l'eau du lait condensé, fabriqué de cette matière première, dépasse 23,5 p. 100.

3) La condition d'hydrolyse du lactose qui peut être considérée comme la plus favorable, c'est l'incubation à $8^{\circ} \mathrm{C}$ pendant $15 \mathrm{~h}$. L'usage de températures plus élevées provoque des défauts de goût et d'odeur dans le lait hydrolysé et ensuite dans le produit fini.

\section{R és u m é}

Le rapport tend à établir les conditions optimales d'hydrolyse enzymatique du lactose dans le lait. La décomposition partielle du lactose dans le lait de transformation peut prévenir la formation de la texture sableuse dans le lait condensé sucré. L'hydrolyse est 
effectuée en ajoutant 0,1 p. 100 de $\beta$-D-galactosidase préparée à partir de la levure Saccharomyces fragilis. Les meilleures conditions pour l'hydrolyse, sans effets occasionnels indésirables, ont été trouvées à $8^{\circ} \mathrm{C}$ pendant $15 \mathrm{~h}$. Pour obtenir l'effet désiré, il suffit d'hydrolyser 15 p. $100 \mathrm{du}$ lactose présent dans le lait. La méthode qui consiste à refroidir le produit après fabrication peut influencer aussi d'une manière définie le nombre et la dimension des cristaux de lactose formés. Le refroidissement rapide et l'agitation intensive sont des facteurs additionnels qui permettent d'éviter le défaut de la texture sableuse dans le lait condensé sucré.

\section{S u m m ary}

Studies were made to determine optimum conditions for hydrolysis of milk lactose. A partial break down of lactose in processing milk could prevent the defect of sandiness in condensed sweetened milk. The hydrolysis was carried out by adding 0.1 p. 100 of $\beta$-D-galactosidase which had been prepared from yeast Saccharomyces fragilis. The most favorable conditions for hydrolysis, with no undesirable side effects, were found to be at $8^{\circ} \mathrm{C}$ for 15 hours. The hydrolysis of about 15 p. 100 lactose apeared to be sufficient for obtaining a desirable effect. The way of cooling the product after manufacture was found to influence a number and a size of lactose cristals formed in definite manner. A rapid cooling and intensive agitation are additional factors which eliminate the defect of sandiness from condensed sweetened milk.

\section{Bibliographie}

[1] Budslawskr (J.) (1963). - Chemia i analiza mleka oraz jego przetworow. PWRiL, Warszawa.

[2] Dluzewski (M.) (1969). - Studia nad laktoza i jej zastosowaniem w przemysle. Roczn. Techn. i Chemii Zywn. 16, 21.

[3] Hunziker (O.) (1949). - Condensed milk and milk powder. Published by author Le Grange, Illinois.

[4] Praca Zbiorowa (1967). - Metody badania zywnosci (wg norm). PWRiL, Warszawa.

[5] PiJanowski (E.) (1971). - Zarys chemii i technologii mleczarstwa. PWRiL, Warszawa.

[6] Pomeranz (I.) (1964). - Lactase ( $\beta$-D-galactosidase). I. Occurence. and properties. II. Possibilities in food industries. Food Technol., 18, 682.

[7] Sparks (I. B.) (1937). - The Crystallization of Lactose. Volume Jubiliare. Louis E. C. Dopples, Geneva (Switzerland).

[8] Stimpson (E. G.) (1954). - Frozen concentrated milk products. U.S. Patent, 266,8765 .

[9] Wendorf (W. L.), AMundsen (C. H.) (1971). - Characterization of $\beta$-D-galactosidase from Saccharomyces fragilis. J. Milk Food Technol., 34, 300. 